\title{
Factores de Riesgo en mujeres embarazadas, infectadas por Toxoplasma gondii en Armenia- Colombia
}

\begin{abstract}
Christian A. López-Castillo1, Julieth Díaz-Ramírez2 y Jorge E. Gómez-Marín3 ${ }^{1}$ Estudiante de Medicina. Grupo de Estudio en Parasitología Molecular (GEPAMOL). Centro de Investigaciones Biomédicas. Universidad del Quindío. Av. Bolívar 12N, Armenia (Quindío), Colombia, Sur América. Tel/Fax +57 67 460168. E-mail: salomon_tauber@yahoo.es ${ }^{2}$ Estudiante de Medicina. Grupo de Estudio en Parasitología Molecular (GEPAMOL). Centro de Investigaciones Biomédicas. Universidad del Quindío. Av. Bolívar 12N, Armenia (Quindío), Colombia, Sur América. Tel/Fax +57 67 460168. E- mail: judira@hotmail.com

${ }^{3}$ Médico. MSc. Biomedicina Tropical PhD. Biología Parasitaria, Post-doctorado Espectroscopía Biomolecular. Director Grupo de Estudio en Parasitología Molecular (GEPAMOL). Centro de Investigaciones Biomédicas. Universidad del Quindío. Av. Bolívar 12N, Armenia (Quindío), Colombia, Sur América. Tel/Fax +57 67460168.

E-mail: gepamol2@uniquindio.edu.co
\end{abstract}

Recibido 1 Abril 2005/Enviado para Modificación 2 Mayo 2005/Aceptado 27 Mayo 2005

\section{RESUMEN}

Objetivos Identificar los factores de riesgo para toxoplasmosis aguda en mujeres embarazadas del municipio de Armenia.

Métodos Estudio de casos y controles en 14 casos y 34 controles (promedio de edad $23 \pm 5,5$ y $23 \pm 5,1$ respectivamente; $p=0,93$ ). Participaron mujeres embarazadas con criterios diagnósticos para infección aguda o mujeres con hijos infectados por Toxoplasma gondii y mujeres embarazadas negativas para IgG específico para Toxoplasma.

Resultados Los factores de riesgo más fuertemente asociados fueron carne poco cocida (OR: 13,2, IC95 \% 1,3-132, p=0,01), tomar bebidas hechas con agua sin hervir (OR: 4,5, IC95 \% 1,1-17 $p=0,01$ ) y el contacto con gatos menores de 6 meses (OR Indefinido, $p=0,01$ ). Tomar agua embotellada fue un factor protector (OR: 0,24, IC95 \% 0,06-0,95, p=0,02).

Conclusión El $42 \%$ de las infecciones por Toxoplasma gondii en Armenia estuvieron asociadas al contacto con gatos y el consumo de carne poco cocida. Se sugiere también recomendar el consumo de agua de bolsa como una medida protectiva para toxoplasmosis en el embarazo.

Palabras Clave: Toxoplasma, toxoplasmosis, factores de riesgo, epidemiología, embarazadas, Colombia (fuente: DeCS, BIREME) 


\section{ABSTRACT \\ Risk factors for Toxoplasma gondii infection in pregnant women in Armenia, Colombia}

Objectives To determine the risk factors associated with acute toxoplasmosis during pregnancy in Armenia.

Methods Case-control study; 14 cases and 34 controls (mean ages $23 \pm 5.5$ and $23 \pm 5.1$ respectively; $p=0.93$ ). Cases were pregnant women with serological criteria for acute toxoplasmosis and controls were seronegative pregnant women for Toxoplasma IgG.

Results The risk factors more strongly predictive of acute toxoplasmosis in pregnant women were: eating undercooked meat (OR: 13.2; 95\% Cl: 1.3132; $p=0.01$ ), drinking beverages prepared with un-boiled water (OR: 4.5; 95\% Cl: 1.1-17; $\mathrm{p}=0.01$ ), and contact with cats aged less than 6 months (OR: undefined, $p=0.01$ ). Drinking of bottled water was a protective factor (OR: 0.24; 95\% Cl: 0.06-0.95; $p=0.02$ ).

Conclusions $42 \%$ of Toxoplasma gondii infections in pregnant women Armenia were associated to contact with young cats and to consumption of undercooked meat. Drinking of bottled water to prevent toxoplasma infection during pregnancy is also recommended.

Key Words: Toxoplasma, toxoplasmosis, risk factors, epidemiology, pregnancy, Colombia (source: $M e S H, N L M$ )

L

a toxoplasmosis en una enfermedad parasitaria altamente prevalente en el mundo y cuando es adquirida por primera vez en el embarazo puede causar serios daños en el feto. Las consecuencias en el niño pueden ser tan diversas como hidrocefalia, microcefalia, calcificaciones cerebrales, coriorretinitis, o terminar en un aborto, dependiendo de la fecha en que la madre se infectó. La infección puede ser adquirida de varias formas, principalmente por vía oral a través del consumo de ooquistes excretados por el gato o quistes tisulares en carne mal cocida. Entre los medios para la ingestión de estos se han identificado los vegetales mal lavados y el contacto directo con tierra; aunque en un alto porcentaje de casos no se identifica la infección. También han sido estudiados factores como el contacto con gatos, con perros, con cerdos, con consumo de leche o huevos, con pobre higiene, con consumo de vegetales crudos, con bajo nivel socioeconómico, habitar en área rural o realizar ocupaciones que impliquen contacto con suelo (1-15). Cada uno de estos factores en algunos estudios se ha encontrado relación, mientras en otros dicha asociación no se ha encontrado (1-15).

En Colombia, en los últimos 12 años se han realizado numerosos estudios seroepidemiológicos de la toxoplasmosis adquirida durante el embarazo. Según el estudio nacional de salud de 1980 la tasa de positividad es de 47,1 \%, 
con títulos altos predominantemente en la zona Atlántica. En este mismo estudio el 1,8\% de las mujeres embarazadas presentó títulos altos de anticuerpos, lo cual es probablemente indicativo de infección reciente (16). Particularmente en el Departamento del Quindío, se han reportado tasas entre 0,7 y $1,6 \%$ de gestantes con marcadores serológicos de infección aguda (17). En el municipio de Armenia, la prevalencia de la toxoplasmosis es del $60 \%$, constituyéndose esta parasitosis en una prioridad en salud pública en la localidad (17). De acuerdo con estas cifras, en el Quindío se calculaban 30 a 120 nuevos casos en embarazadas en 1997 (18), siendo evidentes los altos costos que representa esta entidad para nuestra sociedad.

A pesar de ser un problema de salud pública en Colombia, solamente la ciudad de Armenia en Colombia realiza un programa de tamizaje para la toxoplasmosis congénita durante el embarazo. Desde el 2000 a la fecha, cada año aproximadamente 600 madres son analizadas y 2 a 5 casos de toxoplasmosis congénita son detectados.

En el Quindío un estudio realizado en 937 gestantes demostró que preguntas simples como: ¿tiene gatos en la casa ahora? o ¿acaricia o duerme con su gato?, no identificaban las madres con riesgo, mientras que la pregunta ¿consume carne cruda?, identificó una población con 2,5 veces más riesgo para la infección reciente y la pregunta ¿tiene ganglios o fiebre? Identificó una población con 4,5 veces más riesgo. Sin embargo esa encuesta de factores de riesgo solo identificó al 33 \% de las gestantes con infección reciente quedando sin identificar la mayor parte de las embarazadas (18).

Estudios recientes han asociado la infección aguda con consumo de agua no tratada o del acueducto $(19,20)$. En Julio de 1997, en la investigación de un brote de toxoplasmosis en Columbia Britanica (Canadá) se observó al realizar un mapeo de los casos que existía una asociación significativa entre la infección aguda y la residencia en el área de un sistema de distribución de agua (19). En un estudio en Brasil en Enero de 2003 (20) se encontró que beber agua no filtrada incrementaba el riesgo de seropositividad en personas de grupo socioeconómico bajo (Odds Ratio [OR]: 3.0, intervalo de confianza [IC] 95 \%: 1.3 a 6.9) y medio (OR: 1.7, IC $95 \%$ : 1.2 a 2.3). Este estudio se realizó sobre una población seleccionada aleatoriamente de escuelas, un batallón y la comunidad de Campos dos Goytacazes (Estado de Rió de Janeiro) en donde el $79 \%$ de la población es de los grupos socioeconómicos medio y bajo, y se observó que el 26,9 \% de los encuestados consumía agua no filtrada en su hogar y el 36,8 \% consumía por lo menos un poco de agua sin filtrar. En América Latina esta importante asociación solo se ha estudiado en esta comunidad de Brasil, por lo que es necesario adelantar investigaciones 
que confirmen estas observaciónes (20). El grupo de Parasitología de Reims (Francia) propone la detección en agua del parásito utilizando PCR e inoculación en ratón para determinar la presencia de infección. Comparando la técnica molecular y la inoculación, la PCR fue más sensible siempre (21, 22).

Lo anterior indica la potencial importancia de la transmisión de ooquistes a través del agua, factor que representaría un objetivo para intervenir en la prevención de la enfermedad y como un factor a tener en cuenta al evaluar el riesgo de infección de una gestante, además de dilucidar un poco más los mecanismos de adquisición de la infección.

Los objetivos del presente trabajo fueron identificar los factores asociados a la infección por Toxoplasma gondii en embarazadas en el municipio de Armenia, valorando especialmente el papel del agua como vector del parásito utilizando un diseño de estudio de casos y controles.

\section{MATERIALES Y MÉTODOS}

\section{Población estudiada}

La ciudad de Armenia tiene 316301 habitantes, 97 \% son urbanas y la población obstétrica es de 6636 mujeres. El porcentaje de madres que son positivas anualmente por IgM anti-Toxoplasma está entre 1,3 \% y 2,3 \% (18).

Diseño del estudio

Utilizamos un diseño de casos y controles en mujeres que estuvieron embarazadas, que residían en el municipio de Armenia y que fueron atendidas en el programa de toxoplasmosis del Centro de Investigaciones Biomédicas de la Universidad del Quindío de Armenia, entre Septiembre del 2004 y Febrero del 2005.

Cálculo de la muestra

Para el cálculo de la muestra la proporción caso-control fue de 1:2. Se tomaron 14 casos y se calcularon (Epi-Info 2004) 28 controles para obtener un confiabilidad del $95 \%$ y un poder del $80 \%$, partiendo de una prevalencia de exposición al potencial factor de riesgo del 60 \% y un OR estimado de 9,61.

Definición de casos 
Los casos fueron mujeres con IgG mas IgM y/o IgA anti-Toxoplasma positivas durante el embarazo o con recién nacido con toxoplasmosis congénita confirmada por pruebas serologicas o prueba de reacción en cadena de la polimerasa (PCR) especifica para el gen B1 de Toxoplasma en liquido amniótico o sangre, realizada como fue descrito previamente (23). Todos los casos tenían residencia en el municipio de Armenia, Quindío.

Definición de controles

Los controles fueron mujeres de edades, fecha de embarazo y lugar de residencia similares, que hubieran tenido IgG anti-Toxoplasma negativa durante el embarazo y en el momento de realizar el estudio.

Recolección de la información

Para la recolección de la información se utilizó una encuesta, diligenciada durante visitas domiciliarias, llamadas telefónicas realizadas por los investigadores y durante la consulta especial de toxoplasmosis realizada en la Universidad. Esta encuesta se enfocó en la exposición a factores de riesgo para infección durante el embarazo ya reconocidos como el consumo de carne medio cocida, comer en restaurantes, tener gatos en la casa y contacto con gatos menores de 6 meses de edad; y el tipo de agua que consumía durante el embarazo. Se incluyeron preguntas como procedencia del agua de la casa; si tomaban agua filtrada o de ozono; si tomaban agua de botella bolsa o botellón; si durante el embarazo tomaron alguna vez agua sin hervir; si alguna vez tomaron bebidas hechas con agua sin hervir; si bebieron agua directamente de ríos o quebradas y si tomaron agua directamente de la llave.

Pruebas serológicas

Las muestras de suero fueron analizadas por el Centro de Investigaciones Biomedicas de la Universidad del Quindio para toxoplasmosis usando un estuche de inmunoensayo (ELISA DSL-05-10-TXG para IgG y DSL-05-10TXM para IgM) para anticuerpos específicos anti-Toxoplasma (Diagnostic Systems Laboratories, Inc., Webster, TX, USA). En el programa de control todas las madres positivas para IgG se les realizaban analisis para IgM. Las positivas para IgM se confirmaban en algunas con la prueba de IgM e IgA por western blot utilizando el estuche comercial ID Blot Toxoplasma de DPC Laboratorios (EUA).

Análisis estadístico 
Se utilizaron las variables obtenidas en la encuesta, se relacionaron en una base de datos (Excel 8.0). Luego los datos fueron analizados de manera individual y en relación con la infección, se calculó la razón de disparidad u "Odss Ratio" (OR), los intervalos de confianza de 95 \% y la probabilidad. Para los cálculos se utilizó el programa de análisis estadístico Epi-Info (versión 2004, Centers for Disease Control and Prevention, Atlanta, Georgia, EUA.).

\section{Aspectos Éticos}

Este estudio fue revisado y aprobado por el comité de bioética de la Universidad del Quindio. A todos los participantes se les informó y se obtuvo el consentimiento informado. Se siguieron las normas éticas estipuladas en la resolución 008430 de 1993 del Ministerio de Salud Pública de Colombia.

\section{RESULTADOS}

Inicialmente se seleccionaron 58 mujeres que participaban en el programa de tamizaje prenatal para toxoplasmosis en el embarazo en la ciudad de Armenia, de las cuales 5 no fueron localizadas y otras 5 no cumplieron los criterios de inclusión. Al final, un total de 48 mujeres embarazadas fueron incluidas en el estudio. Los casos fueron 14 mujeres con criterios de infección durante el embarazo, de ellas 5 bebes resultaron infectados y 3 de ellos presentaron síntomas de toxoplasmosis congénita, 1 bebe tenía PCR para el gen B1 de Toxoplasma gondii positivo en sangre y 1 madre fue diagnosticada a través de PCR de líquido amniótico positivo para el mismo gen de T. gondii. De las mujeres que aun no habían dado a luz, 6 presentaban bandas para IgA anti- Toxoplasma por prueba de western blot y a 3 no se les realizó esta prueba. Así mismo 34 mujeres control participaron del estudio quienes al momento de realizar la encuesta estaban embarazadas y su IgG anti-Toxoplasma era negativa. Todas las mujeres vivían en Armenia en el momento del estudio y durante el embarazo, pertenecían a estratos socioeconómicos entre 0 y 3 y el agua de su casa provenía del acueducto.

Los resultados del análisis estadístico de los diferentes factores de riesgo se encuentra relacionado en la Tabla 1. El riesgo de infección por Toxoplasma aumentó en mujeres que reportaron comer carne cruda (el tipo de carne no fue discriminada) o a medio cocer (OR: 13,2, IC95 \% 1,31 132,01, $\mathrm{p}=0.01$ ), beber bebidas hechas con agua sin hervir (OR: 4,5, IC95 \% $1,18-17,79, \mathrm{p}=0,01)$ y tener contacto con gatos menores de 6 meses (OR: indefinido, IC95 \% indefinidos, $\mathrm{p}=0,01$ ). En contraste, beber agua de bolsa, botella o botellón fue un factor protector (OR: 0,24, IC95\% 0,06-0,95, 
$\mathrm{p}=0,02$ ). El beber agua filtrada o de ozono fue hallado solo en 4 gestantes de los controles; sin embargo las diferencias no fueron estadísticamente significativas. En 57,1 \% de los casos y 41,2 \% de los controles (OR:1,9, IC95 \% $0,54-6,71, p=0,16$ ) se encontró que bebían directamente de la llave. Comer en restaurantes se encontró en el 85,7 \% de los casos y en el 73,5 \% de los controles (OR: 2,16, IC95 \% 0,4- 11,58, p =0,19). Ninguno de los casos o controles bebieron agua directamente de ríos o quebradas.

\section{DISCUSIÓN}

Alrededor del mundo se han realizado diferentes estudios de casos y controles que apuntan al consumo de carne cruda como un factor de riesgo muy importante para la infección por Toxoplasma en mujeres embarazadas (3, 7, 12, 15). En el estudio multicéntrico Europeo (3), se reportó que consumir carne a medio cocer explica entre el 30 y el $63 \%$ de las infecciones en diferentes partes del continente europeo. El consumo de carne cruda aumenta el riesgo de infección en Francia 5,5 veces (7) y en Noruega 3,4 veces (15).

Tabla 1. Factores de riesgo para la infección por $T$. gondii Armenia, Colombia (Marzo de 2005)

\begin{tabular}{|c|c|c|c|c|c|c|c|}
\hline \multirow{2}{*}{$\begin{array}{l}\text { Factores de } \\
\text { riesgo }\end{array}$} & \multicolumn{2}{|c|}{ Casos } & \multicolumn{2}{|c|}{ Controles } & \multirow{2}{*}{ OR } & \multirow{2}{*}{$\begin{array}{l}\text { Intervalos de } \\
\text { Confianza }\end{array}$} & \multirow{2}{*}{$p$} \\
\hline & \# & $\%$ & $\#$ & $\%$ & & & \\
\hline $\begin{array}{l}\text { Consumo } \\
\text { agua filtrada }\end{array}$ & 0 & 0 & 4 & 11,8 & 0,00 & Indefinido & 0,11 \\
\hline $\begin{array}{l}\text { Consumo } \\
\text { agua botella } \\
\text { Consumo }\end{array}$ & 4 & 28,6 & 21 & 61,8 & 0,24 & $0,06-0,95$ & 0,02 \\
\hline $\begin{array}{l}\text { agua sin } \\
\text { hervir }\end{array}$ & 10 & 71,4 & 17 & 50 & 2,5 & $0,65-9,55$ & 0,09 \\
\hline $\begin{array}{l}\text { Consumo } \\
\text { bebidas agua } \\
\text { sin hervir }\end{array}$ & 10 & 71,4 & 12 & 35,3 & 4,5 & $1,18-1,79$ & 0,01 \\
\hline $\begin{array}{l}\text { Consumo } \\
\text { agua de la } \\
\text { llave }\end{array}$ & 8 & 57,1 & 14 & 41,2 & 1,90 & $0,54-6,71$ & 0,16 \\
\hline $\begin{array}{l}\text { Ingestión } \\
\text { carne cruda } \\
\text { o a medio } \\
\text { cocer }\end{array}$ & 4 & 28,6 & 1 & 2,9 & 13,2 & $1,31-132,01$ & 0,01 \\
\hline $\begin{array}{l}\text { Visitas a } \\
\text { restaurantes }\end{array}$ & 12 & 85,7 & 25 & 73,5 & 2,16 & $0,40-11,58$ & 0,19 \\
\hline $\begin{array}{l}\text { Contacto con } \\
\text { gatos en } \\
\text { casa }\end{array}$ & 3 & 21,4 & 1 & 2,9 & 9,00 & $0,84-95,68$ & 0,03 \\
\hline $\begin{array}{l}\text { Contacto } \\
\text { gatos }<6 \\
\text { meses }\end{array}$ & 3 & 21,4 & 0 & 0 & Indefinido & Indefinido & 0,01 \\
\hline
\end{tabular}


En nuestro estudio encontramos que el riesgo para infección por Toxoplasma al consumir carne cruda o a medio cocer se aumenta 13,2 veces más (IC95 \% 1,31 - 132,01). Esta variable presentó intervalos de confianza muy amplios, lo que se puede explicar por el tamaño de la muestra, pero mostró el valor de p más significativo.

A diferencia de otros estudios realizados previamente en Armenia y el Quindío, que no encontraron relación con contacto con gatos $(17,18)$, en el presente estudio se encontró que el tener contacto con gatos menores de 6 meses es un factor de riesgo. Esto se puede explicar porque al calcular el OR en los estudios previos se tomaba el resto de la población en el estudio incluyendo los casos crónicos y no se comparo solamente con los porcentajes de prevalencia en seronegativas (18). El contacto con gatos jóvenes aumenta el riesgo de infección de mayor manera que el contacto con gatos mayores, lo que se puede explicar ya que los últimos obtienen inmunidad y la diseminación de ooquistes infectantes por ellos es menor (24).

Aunque este estudio no mostró que beber agua directamente de la llave estuviera asociado estadísticamente a un riesgo mayor, se encontró que tomar bebidas con agua sin hervir aumenta el riesgo de infección 4,5 veces (IC95 \% 1,18 - 17,79, p=0,01) y que beber agua de botella, bolsa o botellón, es un factor protector. Esto confirma lo recientemente encontrado en Brasil, que indica la potencial importancia de la transmisión de ooquistes de T. gondii en agua de distribución para consumo por acueducto y en Francia donde un estudio mostró que 1 de 6 muestras de agua pública contenían DNA de Toxoplasma (21). El porqué no se encontró asociación significativa en el presente estudio se puede explicar por el tamaño de la muestra y porque posiblemente la transmisión por agua de acueducto sea un evento cíclico. Esto destaca el papel potencial del agua como vector de la infección en casos no relacionados con otras fuentes conocidas como contacto con gatos y el consumo de carne cruda, hábitos encontrados en un bajo porcentaje de la población: 8,3 y 10,4 \% respectivamente, contra 14,9 - 34 \% y 25,6 - 38,9 \% de otros estudios $(3,20)$, y que se asociaron al $42,8 \%$ de los casos afectados. Esto significa que se puede explicar en por lo menos el $42 \%$ de los casos un origen de la infección (contacto con gatos o consumo de carne poco cocida) en embarazadas de Armenia, quedando un 58 \% factor de riesgo asociado con al encuesta utilizada. La recomendación de beber agua de botella, bolsa o botellón durante el embarazo podría ser medida profiláctica una muy importante. El impacto de esta medida reduciendo el número de casos de toxoplasmosis en el embarazo debería ser evaluado; mas aun teniendo en cuenta 
que se observó como hábito frecuente en la región el consumo de agua sin hervir en un 56\% de la población estudiada.

En conclusión, este estudio encontró que por lo menos el 42 \% de los casos de toxoplasmosis gestacional en Armenia estuvieron asociados a factores de riesgo conocidos tales como el contacto con gatos y el consumo de carne poco cocida, factores hacia los cuales se deben continuar la recomendación a la población de gestantes para evitarlos. Este estudio también sugiere que recomendar el consumo de agua de bolsa o de botellón puede ser una medida protectiva para toxoplasmosis en el embarazo en la ciudad de Armenia. Finalmente se hacen necesarios estudios prospectivos que midan el impacto de medidas tales como ofrecer agua de bolsa o botellón, eventualmente subsidiadas para la población económicamente desfavorecida, y evaluar su costo beneficio como actividad de salud publica dirigida a la población embarazada •

Agradecimientos. A Ximena Murillo, Álvaro José Nieto, Gloria Maria Gil Castaño y Ana Carolina Henao González, por su ayuda con algunas encuestas. A Jhon Carlos Castaño, Alejandra Giraldo y Maria Mercedes González de Schroeder miembros del equipo responsable por parte de la Universidad del Quindío del programa de control en toxoplasmosis de la ciudad de Armenia, que amablemente facilitaron los datos para localizar las madres casos y controles. A la Dirección General de Investigaciones de la Universidad del Quindío por el apoyo económico para la realización del estudio a través del programa de semilleros de investigación.

\section{REFERENCIAS}

1. Dunachie JF, Siim JC, Hutchison WM. Life cycle of Toxoplasma gondii. Br Med J 1969; 4: 806.

2. Frenkel JK, Hassanein KM, Hassanein RS, Brown E. Transmission of Toxoplasma gondii in Panama City, Panama: a five-year prospective cohort study of children, cats, rodents, birds, and soil. Am J Trop Med Hyg. 1995; 53: 458468.

3. Cook AJ, Gilbert RE, Buffolano W, Zufferey J, Petersen E, Jenum PA, Foulon W, Semprini AE, Dunn DT. So|urces of toxoplasma infection in pregnant women: European multicentre case-control study. European Research Network on Congenital Toxoplasmosis. BMJ. 2000; 321: 142-147.

4. De Camargo MC, Antunes CM, Chiari Cde A. Epidemiology of Toxoplasma gondii infection in the municipality of Ribeirao das Neves, MG. I. Importance of domestic animals as sources of $T$. gondii infection in humans. Rev Soc Bras Med Trop. 1995; 28: 211-214. 
5. Hoghooghi-Rad N, Afraa M. Prevalence of toxoplasmosis in humans and domestic animals in Ahwaz, capital of Khoozestan Province, south-west Iran. Am J Trop Med Hyg 1993; 96:163-168.

6. Arias ML, Chinchilla M, Reyes L, Linder E. Seroepidemiology of toxoplasmosis in humans: possible transmission routes in Costa Rica. Rev Biol Trop. 1996; 44: 377-381.

7. Baril L, Ancelle T, Goulet V, Thulliez P, Tirard-Fleury V, Carme B. Risk factors for Toxoplasma infection in pregnancy: a case-control study in France. Scand J Infect Dis. 1999; 31:305-309.

8. Raz R, Nishri Z, Mates A, Sartani G, Hadad N, Reichman N, Miron D, Flatau E. Seroprevalence of antibodies against Toxoplasma gondii among two rural populations in northern Israel. Isr J Med Sci. 1993; 29: 636-639.

9. MacKnight KT, Robinson HW. Epidemiologic studies on human and feline toxoplasmosis. J Hyg Epidemiol Microbiol Immunol. 1992; 36: 37-47.

10. Mittal V, Bhatia R, Singh VK, Sehgal S. Prevalence of toxoplasmosis in Indian women of child bearing age. Indian J Pathol Microbiol. 1995; 38: 143-145.

11. Bonametti AM, Passos Jdo N, da Silva EM, Bortoliero AL. Outbreak of acute toxoplasmosis transmitted thru the ingestion of ovine raw meat. Rev Soc Bras Med Trop. 1996; 30:21-25.

12. Buffolano W, Gilbert RE, Holland FJ, Fratta D, Palumbo F, Ades AE. Risk factors for recent Toxoplasma infection in pregnant women in Naples. Epidemiol Infect. 1996;116: 347-351.

13. Paul M. Potential risk factors for Toxoplasma gondii infection in cases with recently acquired toxoplasmosis. Przegl Epidemiol. 1998;52(4):447-54.

14. Fan CK, Su KE, Chung WC, Tsai YJ, Chiou HY, Lin CF, Su CT, Tsai MC, Chao PH. Seroprevalence of Toxoplasma gondii antibodies among Atayal aboriginal people and their hunting dogs in northeastern Taiwan. Jpn J Med Sci Biol 1998; 51:35-42.

15. Kapperud G, Jenum P. A., Stray-Pedersen B., Melby K., Eskild A., Eng J. Risk Factors for Toxoplasma gondii Infection in Pregnancy. Results of a Prospective Case-Control Study in Norway. Am J Epidemiol. 1996; 4: 405412.

16. Juliao O, Corredor A, Moreno GS. Estudio Nacional de Salud: Toxoplasmosis en Colombia, Ministerio de Salud. Bogotá: Imprenta Instituto Nacional de Salud; 1988.

17. Gómez JE, Castaño JC, Montoya MT. Toxoplasmosis congénita en Colombia: Un problema subestimado de salud pública. Colombia Médica 1995; 26: 66-70.

18. Gomez JE, Castaño JC, Montoya MT. A maternal screening program for congenital toxoplasmosis in Quindío (Colombia) and application of mathematical models to estimate incidence using age-stratified data. Am J Trop Med Hyg. 1997; 57: 180-186.

19. Bowie WR, King AS, Werker DH, Isaac-Renton JL, Bell A, Eng SB, Marion SA. Outbreak of toxoplasmosis associated with municipal drinking water. The BC Toxoplasma Investigation Team. Lancet. 1997; 350:173-177. 
20. Garcia Bahia-Oliveira LM, Jones JL, Azevedo-Silva Juliana. Highly Endemic, Waterborne Toxoplasmosis in North Rio de Janeiro State, Brazil. Emerg Infect Dis. 2003; 9:55-62.

21. Villena I, Aubert D, Gomis P, Ferté H, Inglard JC, Denis-Bisiaux H, Dondon JM, Pisano E, Ortis N, Pinon JM. Evaluation of a strategy for Toxoplasma gondii Oocyst detection in water. Appl. Environ. Microbiol. 2004; 70:4035-4039.

22. Isaac- Renton J, Bowie WR, King A, Irwin S, Ong C, Fung CP, Shokeir O and Dubey JP. Detection of Toxoplasma gondii oocysts in drinking water. Appl Environ Microbiol 1998; 64:2278-2280.

23. Ponce N, Gomez JE. Estandarización y validación clínica de la prueba de reacción en cadena de la polimerasa (PCR) para diagnóstico de toxoplasmosis cerebral en pacientes infectados por el VIH. Infectio 2003; 7: 8-14

24. Frenkel JK, Ruiz A, 1980. Human Toxoplasmosis and cat contact in Costa Rica. Am J Trop Med Hyg 1980; 29:117-118. 